\title{
INSTALACIONES INTELIGENTES EN EL USO RACIONAL DE LA ENERGÍA ELÉCTRICA
}

\author{
Margarita Fredesvinda Murillo Manrique
}

\section{Resumen}

El presente artículo pretende motivar en la práctica el uso racional de la energía eléctrica inicialmente con actividades en las viviendas residenciales, que posteriormente se convertirán en creación de valor que repercutirá en los centros laborales, en las instituciones educativas y en las industrias, considerando que en estas últimas el uso de energía se da en todos sus procesos; tal es así que la filosofia que se practica en la actualidad se resume en el siguiente slogan "Eficiencia Energética + Nuevas tecnologías = Ahorro Energético", lo que hace ya varios años se viene difundiendo en nuestro país; sin embargo aun no ha sido posible disminuir el consumo eléctrico de manera significativa, lo que motivó para realizar esta investigación cuyos resultados obtenidos se presentan en cuadros de consumo de energía en diferentes zonas de Lima, así como las propuestas para que una serie de artefactos de uso cotidiano en las viviendas como en la industria puedan reducir el consumo eléctrico de manera significativa mediante la aplicación de nuevas tecnologías disponibles en el mercado y por consiguiente lograr un ahorro económico importante con la practica del uso racional de la energía eléctrica.

Palabras Clave: Instalaciones inteligentes, eficiencia energética, ahorro energético, uso racional. 


\section{Abstract}

This article aims to encourage the practice of rational use of electricity at home activities initially, which later become value creating impact in the workplace, in educational institutions and industries, in which energy use is given in all processes, so much so that philosophy is practiced today is summarized in the following slogan "Energy Efficiency + New Technology = Energy Savings, which for several years has been spreading and practicing, however has not been possible to reduce the power consumption significantly, prompting for this research and the results obtained are presented in tables of power consumption in different areas of Lima, as well as proposals for a series of artifacts everyday use in homes and industry can reduce power consumption significantly by applying new technologies available in the market and therefore achieve a significant cost savings in the practice of rational use of energy.

Key words: Facilities smart, energy efficiency, energy saving, rational use.

\section{INTRODUCCIÓN}

La electricidad es sin duda uno de los principales factores que impulsa el desarrollo de la sociedad; porque permite optimizar los procesos y acelerar el rendimiento de la industria, mejorando el nivel de vida con sus diferentes aplicaciones.

Con la finalidad de promover una cultura de uso racional y eficiente de la energía, para impulsar el desarrollo sostenible del país, el Ministerio de Energía y Minas estableció la Dirección General de Eficiencia Energética, como un órgano técnico formativo integrado a su nueva estructura orgánica, aprobada por Decreto Supremo $\mathrm{N}^{\circ}$ 026-2010-EM.

Una instalación inteligente es un sistema que permite la comunicación integrada a distancia del usuario desde cualquier punto donde existe conectividad de forma que permita una operación más eficiente de sus sistemas domésticos, racionalizando de manera eficiente el consumo de energía eléctrica.

En ese sentido, todos los esfuerzos que los seres humanos puedan hacer para reducir su consumo de energía sin afectar el desarrollo socioeconómico; así como el esfuerzo que las empresas suministradoras de energía y los gobiernos realicen para sustituir en lo posible los esquemas tradicionales de suministro de energía, basados en el consumo de combustibles fósiles generadores de gases efecto invernadero (GEI), por esquemas de suministro de energía basados en fuentes renovables, muy probablemente evitará, o cuando menos tenderá a reducir los efectos no deseados en la naturaleza y por ende en el propio ser humano.

En este contexto de preocupación mundial, las sociedades demandan cada vez más expertos en áreas de electricidad, transporte y concesiones 
en infraestructura de servicios energéticos. Por ello, es necesario fortalecer las capacidades humanas, aprovechando la gestión del conocimiento, las experiencias exitosas y el avance tecnológico para profundizar los conocimientos en el uso racional de la energía.

\section{MATERIAL Y MÉTODOS}

Como primer paso, se presenta el estado del arte con toda la información relevante relacionada con la instalación eléctrica, su problemática y la justificación de las instalaciones inteligentes. A partir de esa información, se han detectado las tecnologías clave y su implicación en el desarrollo del uso racional de la energía.

\section{Estructura del Sector Eléctrico}

En el sector eléctrico se gestiona uno de los servicios básicos y fundamentales para todo desarrollo productivo, económico y social de un país. Por esta razón, es preponderante la promoción de inversiones que provean un servicio eléctrico seguro, continuo, de buena calidad y que permita la ampliación de la cobertura eléctrica nacional.

Actualmente, en el Perú, las oportunidades de inversión se pueden encontrar en las tres actividades que conforman la integración vertical del negocio eléctrico; la generación, transmisión y distribución.

En dicho esquema de negocio, el sector eléctrico peruano ha desarrollado una organización que promueve la competencia y la inversión y que además es reconocido regionalmente por tener un modelo regulatorio moderno que ha evolucionado oportunamente ante los desafios de índole económico, social, ambiental y climatológico.

Figura $N^{\circ} 01 .-$ Sistemas de Generación, Transmisión y Distribución hacia Usuarios Finales

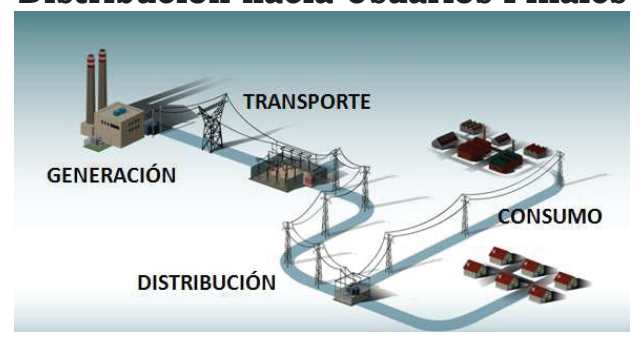

Actualmente, seguimos usando una red eléctrica que fue desarrollada hace bastante tiempo, sin embargo, en un fututo inmediato deberemos afrontar nuevos desafios que surgen de la liberalización de los mercados y de la evolución de la tecnología en este campo.

Aunque el funcionamiento de las redes actuales es correcto, se debe trabajar para proporcionar un suministro eléctrico seguro y sostenible. Las nuevas especificaciones que implementará el sector eléctrico serán las siguientes.

- Participación activa del usuario: En la red actual el usuario es una parte completamente pasiva, el desarrollo de las redes pretenderá desarrollar una actuación del usuario más participativa, surgiendo oportunidades de micro generación, demanda energética flexible, servicios adaptados a sus necesidades, etc. 
Para lograr este punto será necesario incentivar la participación del usuario a la hora de entregar energía generada localmente, en función de su cantidad y la franja horaria.

- Automatización de la red eléctrica: Esto permitirá realizar un mantenimiento mucho más eficiente de todos los componentes de la red, incluso implementando soluciones de gestión remota. Por tanto, será necesario realizar una fuerte inversión en la renovación de las infraestructuras existentes. Existen proyectos que tratan sobre estos conceptos exponiendo una arquitectura basada en IED's, según la norma IEC 61850.

- Seguridad en la generación centralizada: El crecimiento de la red y su capacidad de generación obligará a renovar las centrales generadoras con el fin de asegurar un suministro seguro. De esta forma se mejorará la fiabilidad en el suministro eléctrico ante cualquier perturbación.

- Generación distribuida y fuentes de energía renovable: Gestión energética local, reducción de pérdidas y emisiones, integración en redes de potencia.

- Interoperabilidad con las redes eléctricas europeas: Será necesario mejorar el transporte a largas distancias y la integración de fuentes de energía renovable, fortaleciendo la seguridad europea de suministro a través de unas capacidades de transferencia mejoradas.
- Gestión de la demanda: Desarrollo de estrategias para la regulación local de la demanda y control de cargas mediante medición electrónica y sistemas automáticos de gestión de medidas.

\section{Tecnologias de Transmisión y Distribución, claves para la eficien- cia energética}

La energía eléctrica generada por las centrales se suministra a los usuarios finales, situados a cientos o miles de kilómetros, a través de una red de cables de transmisión y distribución interconectados. Entre los componentes básicos de esta red están las torres de transmisión, conductores/cables, transformadores, interruptores, condensadores / reactores, dispositivos HVDC/FACTS (high-voltage direct / Flexible AC Transmission Devices) y equipos de supervisión, protección, y control. En general, la red que transmite energía a largas distancias, desde las centrales eléctricas hasta las subestaciones próximas a los núcleos de población, se denomina red de transmisión masiva de energía eléctrica y opera a altas tensiones. El sistema de distribución, que entrega energía desde la subestación hasta los usuarios finales, a distancias más cortas, está menos interconectado y opera con tensiones más bajas. El sistema de transmisión y distribución (T+D) se diseña para garantizar una operación fiable, segura y económica de la entrega de energía, sujeta a la demanda de la carga y a limitaciones del sistema.

La "red inteligente" creará energía renovable que es más confiable, flexi- 
ble, segura y eficiente. Uno de los retos de la generación de energía renovable, como la eólica y la energía solar, es que puede ser interrumpida y esta variabilidad afecta la estabilidad de la energía producida. Los dispositivos FACTS direccionan la capacidad de la red y la estabilidad, conforme más energía renovable es puesta en línea.

Las redes de energía están enfrentando una transformación mayor, dirigidas por la necesidad de integrar energía renovable, mejorar la eficiencia de la energía y permitir a los consumidores mayor control sobre el consumo de su energía.

\section{Figura $N^{\circ}$ 02.- Futuro de los Sistemas}

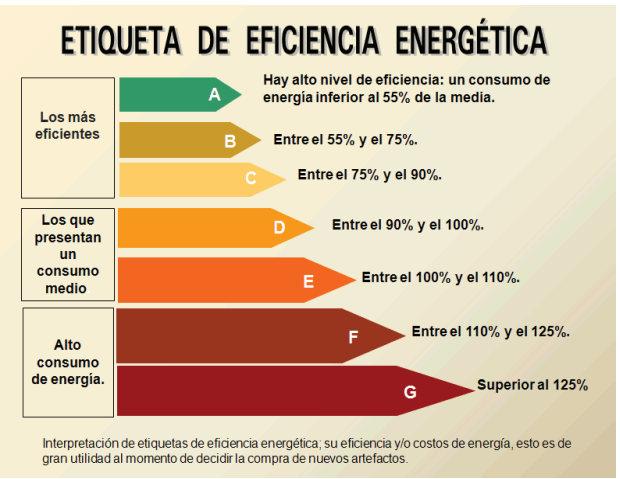

\section{Sistema de Utilización.}

El sistema de utilización está reglamentado por el Código Nacional de Electricidad, Tomo V, en el cual se especifican los requisitos a considerar en las instalaciones eléctricas, tales como diseño, protección, métodos $\mathrm{y}$ materiales, condiciones y pruebas para una eficiente instalación; así mismo, especifica las recomendaciones para los sistemas de comunicación.
A medida que evoluciona, la "red inteligente" combinará tecnologías de potencia establecidas con analítica avanzada, dispositivos inteligentes y tecnologías de automatización, para crear un sistema de energía que sea más confiable, flexible, seguro y eficiente, y tener un menor impacto en el ambiente.

La tecnología FACTS como los compensadores Static Var y la serie de capacitores, permiten fluir más energía a través de las líneas de energía existentes, mientras mejoran estabilidad en el voltaje y también crean una red de energía más resistente a las oscilaciones del sistema y las perturbaciones.

\section{de Transmisión y Distribución}

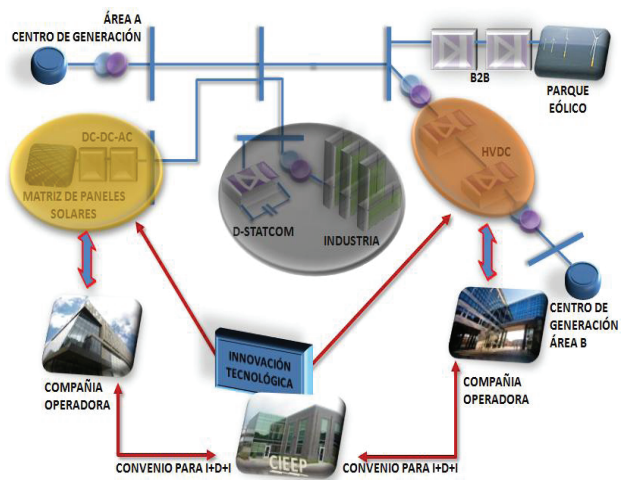

Así también, este Tomo tiene por objeto establecer las prescripciones consideradas necesarias para la seguridad de las personas y de la propiedad frente a los peligros que aparecen por el uso de la electricidad, para lo cual cubre los conductores y equipos eléctricos que operan, o están destinados a operar, a cualquier tensión en instalaciones eléctricas para edificaciones públicas o privadas, construcciones y predios. 
Las instalaciones eléctricas constituyen uno de los elementos importantes en las construcciones domiciliarias; motivo por el cual el tema es de permanente interés, $y$, considerando los cambios tecnológicos recientes, han modificado muchos los materiales y procedimientos usados para los proyectos de construcción de las instalaciones eléctricas. Por ello, es conveniente hacer una revisión del tema que al mismo tiempo incluya los cambios en la norma nacional de electricidad.

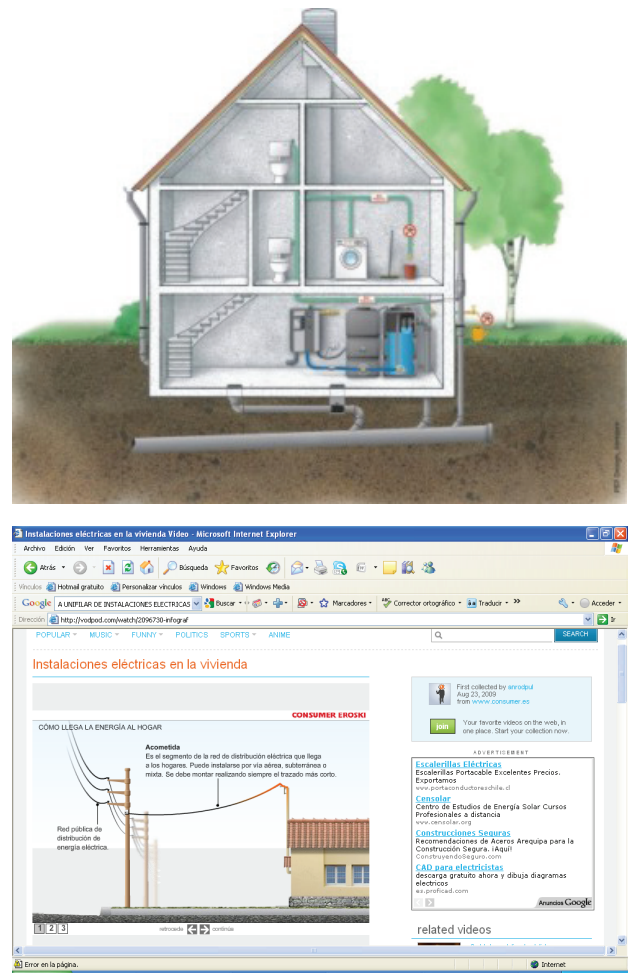

\section{Consideraciones Experimentales}

La realidad es que hoy por hoy la infraestructura de red eléctrica existente parece que cumple parte de las expectativas (desde la generación tradicional y renovable, hasta el transporte y parte de la distribución) pero tiene que mejorar notablemente desde el punto de vista del usuario final y las funcionalidades que se espera de ella.

Las previsiones indican un crecimiento notable de la demanda, un fuerte incremento de las energías renovables y una necesidad de potencia firme y flexible. Todo ello está haciendo que aparezca un nuevo concepto de red eléctrica, las redes inteligentes, cuya definición básica puede corresponder a "Las redes inteligentes", como las redes eléctricas que pueden integrar de manera inteligente el comportamiento y las acciones de todos los actores conectados a ellas (quienes generan electricidad, quienes la consumen y quienes realizan ambas acciones) para proporcionar un suministro de electricidad seguro, económico y sostenible.

\section{a. Máxima Demanda (M.D)}

Para los cálculos de la Máxima Demanda del cuadro de cargas y para el dimensionamiento del alimentador (cable) del medidor (Kwh) al tablero general (TG), se consideran las siguientes formulas:

Para una red de baja tensión se tiene: $\mathbf{V}=\mathbf{2 2 0}$ Vol

MD $=$ Pot $_{\text {Nom }}=\mathbf{V}^{*} \mathbf{I}_{\text {Nom }}(\mathrm{Kw})$ ecuación 1

$\mathbf{I}_{\text {Nom }}=\mathrm{MD} / 220$ (Amperios) ecuación 2 
Es importante considerar un 25\% más de capacidad para el cálculo del alimentador. Entonces, la corriente de diseño se evalúa considerando:

$\mathbf{I}_{\text {diseño }}=1.25^{*} \mathrm{I}_{\text {Nom }}$ (Amperios) ecuación 3

De esta manera, para el cálculo de corrientes que resulten de cargas expresadas en watts o voltamperes, alimentadas por sistemas de corriente alterna de baja tensión, se deben emplear las tensiones nominales de 220 V o 380 V, según corresponda; o cualquier otra tensión nominal dentro del rango de baja tensión de $1000 \mathrm{~V}$ o menos, que sea aplicable.

Factor de utilización (fu): $\mathbf{f}_{\mathrm{u}}=\frac{P c}{P n}$

Donde: $\mathrm{Pc}=$ potencia consumida en watts

$\mathrm{Pn}=$ potencia nominal en watts

b. Utilización de Factores de Demanda

El dimensionamiento de conductores e interruptores deben ser los mínimos a emplear; salvo que a las dimen- siones normalizadas inmediatamente inferiores, corresponda una capacidad hasta $5 \%$ menor que la calculada, en cuyo caso se pueden utilizar estos conductores e interruptores.

En cualquier caso diferente al de una acometida calculada según las Reglas 050- 200 y 050-202, cuando el diseño de una instalación se base en requerimientos superiores a los dados en esta Sección, las capacidades de acometidas y alimentadores deben incrementarse correspondientemente.

Cuando se instalen 2 o más cargas, de las cuales sólo una pueda ser utilizada a la vez, se debe considerar la carga mayor en el cálculo de la demanda.

La capacidad de los conductores de alimentadores y circuitos derivados debe estar de acuerdo con las Secciones del Código, que traten sobre los respectivos equipos a ser alimentados. Para estos cálculos, considerar la tabla $\mathrm{N}^{\circ} 1$.

Factor de Demanda (fd): Cuantifica la permanencia de la carga durante un periodo de tiempo; se determina por tablas, dependiendo del tipo de carga. 
Tabla $N^{\circ}$ 1.- Watts por metro cuadrado y factores de demanda para acometidas y alimentadores para predios según tipo de actividad

\begin{tabular}{|l|c|c|c|}
\hline \multirow{2}{*}{ Tipo de Actividad } & \multirow{2}{*}{ Watts* m2 } & $\begin{array}{c}\text { Factores de Demanda (\%) } \\
\text { Conductores- } \\
\text { Acometida }\end{array}$ & Alimentadores \\
\hline Bodegas & & 100 & 100 \\
\hline Oficinas: & 30 & & 100 \\
\hline Primeros 930m2 & & 90 & 90 \\
\hline Sobre 930m2 & 50 & 70 & 100 \\
\hline Industrial- Comercial & 50 & 100 & 100 \\
\hline Iglesias & 25 & 100 & 90 \\
\hline Edificios de almacenaje & 10 & 70 & 95 \\
\hline Teatro & 5 & 75 & 100 \\
\hline Auditorio & 30 & 80 & 100 \\
\hline Bancos & 10 & 100 & 100 \\
\hline Clubes & 25 & 90 & \\
\hline
\end{tabular}

c. Grados de electrificación en inmuebles

Se establece el grado de electrificación de un inmueble a efecto de determinar, en la instalación, el número de circuitos y los puntos de utilización que deberán considerarse como mínimo. Los grados de electrificación son:

- Electrificación mínima.

- Electrificación media.

- Electrificación elevada.

Tabla $\mathbf{N}^{\circ}$ 2.- Demanda de potencia máxima

\begin{tabular}{|l|c|c|}
\hline \multicolumn{1}{|c|}{$\begin{array}{c}\text { Grado de } \\
\text { electrifica- } \\
\text { ción }\end{array}$} & $\begin{array}{c}\text { Demanda } \\
\text { de potencia } \\
\text { máxima si- } \\
\text { multanea }\end{array}$ & $\begin{array}{c}\text { Limite de } \\
\text { aplicación }\end{array}$ \\
\hline Mínima & $\begin{array}{c}\text { hasta } 3000 \\
\text { V.A. }\end{array}$ & $\begin{array}{c}\text { hasta } 60 \\
\mathrm{~m}^{2}\end{array}$ \\
\hline Media & $\begin{array}{c}\text { hasta } 6000 \\
\text { V.A. }\end{array}$ & $\begin{array}{c}\text { hasta } 150 \\
\mathrm{~m}^{2}\end{array}$ \\
\hline Elevada & hasta 6000 & Mas de 150 \\
& V.A. & $\mathrm{m}^{2}$ \\
\hline
\end{tabular}

Los picos se suelen producir en franjas horarias donde todos los consumidores acceden simultáneamente a sus electrodomésticos, aunque en general no sean conscientes de ello. La posibilidad de conocer en tiempo real el coste de la energía por parte del usuario final, hace que pueda elegir cuándo utilizar determinados electrodomésticos, repercutiendo en el coste final de sus facturas y reduciendo los picos de consumo.

\section{Gráfico $\mathbf{N}^{\circ}$ 01- Diagrama de carga promedio para una Muestra}

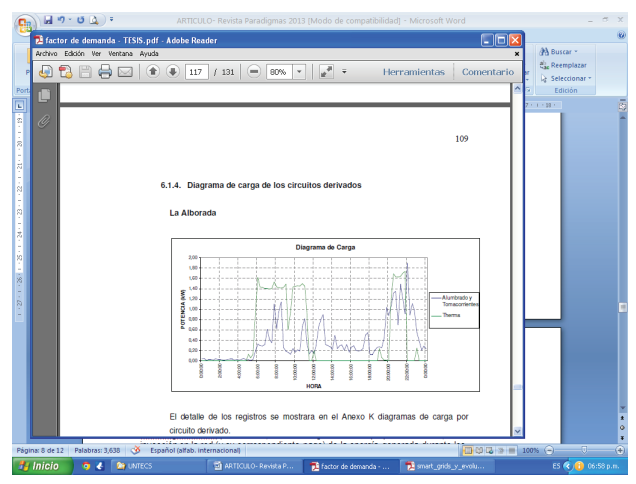

Para ello sería necesario disponer de contadores inteligentes en todos los puntos de consumo, principalmente usuarios domésticos. 
Tabla No 03- Cuadro de cargas para una Muestra Representativa

\begin{tabular}{|c|c|c|c|c|c|c|}
\hline $\begin{array}{c}\text { TABLE- } \\
\text { RO }\end{array}$ & DESCRIPCIÓN & $\begin{array}{l}\text { POTEN- } \\
\text { CIA KW }\end{array}$ & $\begin{array}{c}\text { CANTI- } \\
\text { DAD }\end{array}$ & $\begin{array}{c}\text { CARGA } \\
\text { INSTALADA } \\
(\mathbf{k w})\end{array}$ & F.P. & $\begin{array}{c}\text { MÁXIMA } \\
\text { DEMANDA } \\
\text { (kw) }\end{array}$ \\
\hline \multirow{17}{*}{ TD1 } & $\begin{array}{l}\text { Lámpara Fluores- } \\
\text { cente de } 32 \mathrm{~W}\end{array}$ & 0,032 & 8 & 0,256 & 0,8 & 0,2048 \\
\hline & $\begin{array}{ll}\text { Foco ahorrador } \\
20 \mathrm{~W}\end{array}$ & 0,02 & 15 & 0,3 & 0,8 & 0,24 \\
\hline & $\begin{array}{l}\text { Lámpara tipo Spot } \\
\text { Light } 100 \mathrm{~W}\end{array}$ & 0,1 & 3 & 0,3 & 0,8 & 0,24 \\
\hline & $\begin{array}{l}\text { Bomba de Agua } 0,8 \\
\text { HP }(0,6 \mathrm{Kw})\end{array}$ & 0,6 & 1 & 0,1 & 0,8 & 0,08 \\
\hline & Terma $1500 \mathrm{~W}$ & 1,5 & 1 & 1,5 & 1,0 & 1,5 \\
\hline & $\begin{array}{l}\text { Equipo de sonido } \\
300 \mathrm{~W}\end{array}$ & 0,3 & 3 & 0,9 & 0,8 & 0,72 \\
\hline & TV $100 \mathrm{~W}$ & 0,1 & 3 & 0,3 & 0,8 & 0,24 \\
\hline & DVD $35 \mathrm{~W}$ & 0,035 & 3 & 0,105 & 0,8 & 0,084 \\
\hline & $\begin{array}{l}\text { Refrigeradora } \\
\text { 350w }\end{array}$ & 0,35 & 1 & 0,35 & 0,8 & 0,28 \\
\hline & Lavadora $500 \mathrm{~W}$ & 0,5 & 1 & 0,5 & 0,8 & 0,4 \\
\hline & Licuadora $300 \mathrm{~W}$ & 0,3 & 1 & 0,3 & 0,8 & 0,24 \\
\hline & Microondas 2Kw & 2 & 1 & 2 & 0,8 & 1,6 \\
\hline & Olla Arrocera $1 \mathrm{Kw}$ & 1 & 1 & 1 & 1.0 & 0,8 \\
\hline & Plancha $1 \mathrm{Kw}$ & 1 & 1 & 1 & 0,8 & 0,8 \\
\hline & Ventilador $50 \mathrm{~W}$ & 0,05 & 2 & 0,1 & 0,8 & 0,08 \\
\hline & PC completa $300 \mathrm{~W}$ & 0,3 & 1 & 0,3 & 0,8 & 0,24 \\
\hline & Laptop $150 \mathrm{~W}$ & 0,15 & 1 & 0,15 & 0,8 & 0,12 \\
\hline
\end{tabular}

Se efectuaron mediciones a los clientes seleccionados, para obtener la Demanda Máxima del alimentador principal y de los circuitos derivados del tablero general. Tal información permitió obtener la información necesaria para llenar la tabla 03 mostrada.

Mediante encuestas a los clientes, se obtuvo información del equipamiento eléctrico con que cuentan sus viviendas. Este equipamiento nos dio a conocer la carga total instalada, la carga básica y las cargas especiales, según detalle mostrado en la tabla $\mathrm{N}^{\circ}$ 03.
Utilizando las fórmulas y tablas, se obtienen los cálculos para el diseño del tablero, tal como se muestra en el gráfico siguiente.

\section{Gráfico No 02- Diagrama Unifilar de las cargas para una Muestra Representativa}

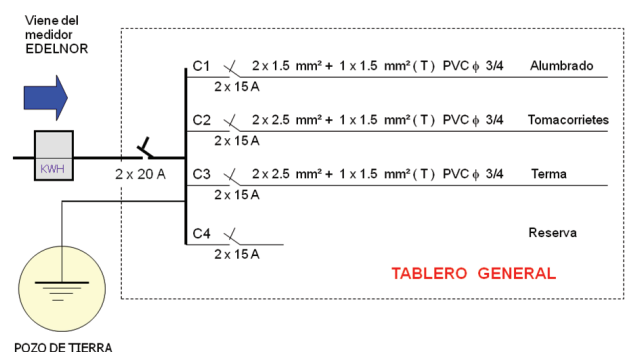


Antes de buscar un plan de autoconsumo energético, el paso principal es saber cuánta energía eléctrica gastamos. Para ello podemos utilizar los cálculos anteriores, o algún tipo de medidor de energía inalámbrico que nos permita conocer nuestro gasto, de tal manera que se deba ir implementando medidas de reducción de consumo y mejor uso de los sistemas eléctricos.

Algunos pasos que se deben seguir para el ahorro de energía:

- Mide cómo y cuánto consumes - Lo que no se mide no se puede mejorar, y todos tenemos en casa equipos electrónicos como ordenadores, DVD, televisores, videoconsolas o cargadores de móvil, que tienen modo stand-by, el cual representa, de media, el $12 \%$ de la factura eléctrica a nivel residencial en el mundo.

- Cámbiate a la climatización eficiente

- Cambia las bombillas

- Optimiza tu contrato de la luz

- Genera tu propia energía limpia

- Consulta a los expertos.

\section{Conclusiones}

La implementación de sistemas automáticos inteligentes puede hacer que las Cias. de electricidad tengan un conocimiento en tiempo real de toda la red, permitiendo una rápida reacción, la detección previa de problemas potenciales y la minimización del impacto de un fallo. Además, si se dispone de una conexión directa con todos los clientes finales, se puede comprobar el estado de sus líneas, consumos y suministros, pudiendo actuar antes de que se produzca el aviso del fallo.

También se ha comentado anteriormente que las infraestructuras que están generando la electricidad actualmente son obras que se han realizado hace muchos años y, por lo tanto, con una tecnología y prestaciones ya muy obsoletas. Así como en el campo de las telecomunicaciones ha habido muchos cambios e inversiones, no lo ha habido en el sector eléctrico, que sigue funcionando con instalaciones y servicios de hace muchos años. Es preciso la inversión en nuevas infraestructuras, que asociados a la reducción de costes de las telecomunicaciones, los avances en sensores y sistemas más inteligentes y procesadores más potentes y rápidos, harán que la generación, distribución y gestión de energía sea más barata, ecológica, y permita ofrecer una nueva serie de nuevos servicios a los usuarios finales.

Para ello, es importante la definición de estándares. Si la infraestructura de las Cias. Eléctricas tiene que interoperar con otros sistemas e industrias (calefacción y aire acondicionado, dispositivos del hogar, como los electrodomésticos, en futuro los coches eléctricos, etc.) debe estar acompañada de estándares que permitan esa interoperabilidad. Todas estas motivaciones lleva a la definición de lo que son las Smart Grids.

Las redes inteligentes integran las tecnologías de la información con las 
infraestructuras eléctricas actuales haciendo una "Internet de la energia" (comunicaciones bidireccionales, flujo multidireccional de la energía y completamente automatizada y controlada).

De todo lo expuesto, se concluye que observando unas simples medidas de manipulación de los elementos con que contamos en nuestros domicilios, podemos realizar un significativo ahorro de energía, que en ocasiones puede superar el 10 por ciento de nuestro consumo.

Si tenemos un consumo bimestral de alrededor de $350 \mathrm{kw} / \mathrm{h}$ y ahorramos más del $10 \%$, al cabo de un año (6 bimestres), prácticamente nos habre- mos evitado abonar a las Cias administradoras de servicios eléctricos un bimestre entero.

\section{NOTAS}

1 Mg. Ing. Electricista. Doctorado en Educación. Docente de la URP. Docente de la UNTECS. Asesora Escuela de Comunicaciones del Ejercito del Perú.

\section{WEB -GRAFÍA}

EDELNOR: Distribución eléctrica. Encontrado el 15-02-2013 en www. edelnor.com.pe

OSINERG: Gerencia Adjunta de Regulación Tarifaria. Encontrado el 15-02-2013 en www.cte.org.pe

MINISTERIO DE ENERGÍA Y MINAS. CNE. Encontrado el 15-022013 en www.mem.gob.pe

\section{REFERENCIAS BIBLIOGRÁFICAS}

DAMMERT, A. GALLARDO, J. \& GARCíA, R. (2005). (Lima- Perú). Reformas Estructurales en el Sector Eléctrico Peruano. Documento de Trabajo $N^{\circ} 5$. Oficina de Estudios Económicos. OSIMERMIN.

ABB. (2007). Perú. Redes eficientes energéticamente. Energía para la eficiencia. Revista ABB 2

SANTACANA, E. ZUCCO, T. \& FENG, X. JIUPING, P. MOUSAVI. M. TANG, L. (2010). España. Tecnologías de transmisión y distribución, claves para la eficiencia energética

FEDIT (centro tecnológico). (2011). España. SMART GRIDS Y LA EVOLUCIÓN DE LA RED ELÉCTRICA. Observatorio Industrial del Sector de la Electrónica, Tecnologías de la Información y Telecomunicaciones. 
